\title{
ASYMPTOTIC EQUILIBRIA IN HOMOGENEOUS AND NONHOMOGENEOUS SYSTEMS
}

\author{
T. F. BRIDGLAND, JR.
}

I. Let $A(t)$ be an $n \times n$ matrix and $p(t)$ an $n$-vector, the elements of each being complex-valued, uniformly bounded and measurable on the real half-line $\{t \mid 0 \leqq t<\infty\}$. Denote by $X(t)$ the (absolutely continuous) fundamental solution matrix, for which $X(0)=I$, of the homogeneous equation associated with the nonhomogeneous system

$$
\frac{d y}{d t}=A(t) y+p(t)
$$

A vector (matrix) will be said to converge if each of its elements tends to a finite limit as $t \rightarrow \infty$; if, for every convergent $p(t)$, every solution of (1) also converges then the coefficient matrix, $A(t)$, of (1) will be said to be of type $(\sim)$. Denoting by $\|\cdot\|$ a vector (matrix) norm, defined as the sum of the moduli of the elements of the vector (matrix), the following characterization of matrices of type $(\sim)$ may be given [1]:

(a) $A(t)$ is of type $(\sim)$ iff $X(t)$ satisfies both

(i) there exist $K>0, \mu>0$ such that $\left\|X(t) X^{-1}(\tau)\right\| \leqq K e^{-\mu(t-r)}$, $t \geqq \tau \geqq 0$, and

(ii) the matrix $Y(t)=\int_{0}^{t} X(t) X^{-1}(\tau) d \tau$ converges.

In the event that both (i), (ii) are satisfied, then for every solution, $y(t)$, of (1),

$$
\lim y(t)=\lim Y(t) \cdot \lim p(t),
$$

where $\lim$ denotes $\lim _{t \rightarrow \infty}$. In [1], it is also shown that if $A(t)$ is "almost constant," that is, if $A(t)=A+B(t)$, where $A$ is a stability matrix $^{1}$ and $B(t)$ satisfies either $\lim \|B(t)\|=0$ or $\int^{\infty}\|B(t)\| d t<\infty$, then $Y(\infty) \equiv \lim Y(t)$ exists and has the value $-A^{-1}$.

In connection with $(\alpha)$, two questions immediately arise, the investigation of which forms the subject of this paper. They are: (a) under what conditions does (ii) imply (i), ${ }^{2}$ and (b) for an arbitrary matrix of type $(\sim)$, what is the value of $Y(\infty)$ ? Complete answers to both these questions are given in the sequel. The author is indebted to $H$. G. Hermes for several valuable suggestions.

Received by the editors January 14, 1963 and, in revised form, May 8, 1963.

${ }^{1}$ A stability matrix is a constant $n \times n$ matrix all of whose characteristic roots have negative real parts.

2 That (i) need not imply (ii) was shown in [1] for the case of a periodic matrix $A(t)$. 
II. The property of type $(\sim)$ matrices expressed in the following lemma will be of fundamental importance in the sequel.

LEMMA. If $A(t)$ is of type $(\sim)$, then $Y(\infty)$ is nonsingular.

Suppose, on the contrary, that $Y(\infty)$ is singular; then there exists a nonzero vector, $c$, in the null space of $Y(\infty)$. Consider the system (1) with $p(t) \equiv c$; it is a consequence of the statement following $(\alpha)$ that $\lim y(t)=Y(\infty) \cdot c=0$. However, if both members of (1) be integrated over the interval $[t, t+1]$, there results

$$
c=y(t+1)-y(t)-\int_{t}^{t+1} A(\tau) y(\tau) d \tau,
$$

from which is obtained, by virtue of the uniform boundedness of $A(t)$, the estimate $\|c\| \leqq(2+N) \sup _{\tau \geq t}\|y(\tau)\|$. Since the right-hand member of this inequality can be made arbitrarily small by taking $t$ large enough, there results $c=0$, a contradiction.

We may now provide an answer to the question (a) above by stating

Theorem 1. The matrix $A(t)$ is of type $(\sim)$ iff $\lim Y(t)$ exists and is the negative of a stability matrix.

For the proof, note first that the nonsingularity of $Y(\infty)$ implies the existence of a $t^{*}<\infty$ such that $Y(t)$ is nonsingular for all $t>t^{*}$. Thus we may define a matrix $T(t)$ by

$$
T(t)=Y^{-1}(t) X(t), \quad t^{\prime} \leqq t,
$$

for arbitrary, but fixed, $t^{\prime}>t^{*}$. Differentiation of (3) leads to

$$
\frac{d T}{d t}=-Y^{-1}(t) T, \quad t^{\prime} \leqq t
$$

which may be written

$$
\frac{d T}{d t}=\left[-Y^{-1}(\infty)+S(t)\right] T, \quad t^{\prime} \leqq t,
$$

where $\lim \|S(t)\|=0$.

When $A(t)$ is of type $(\sim), X(t)$ satisfies (i) and, since $Y^{-1}(t)$ is bounded, it then follows from (3) that $T(t)$ satisfies, for some $L>0$,

$$
\left\|T(t) T^{-1}(\tau)\right\| \leqq L e^{-\mu(t-\tau)}, \quad t \geqq \tau \geqq t^{\prime} .
$$

Armed with this fact, an application to (5) of [1, Theorem 2] and 
its corollary permits the conclusion that $-Y^{-1}(\infty)$, hence $-Y(\infty)$, is a stability matrix.

Conversely, if $-Y(\infty)$ is a stability matrix then an application to (5) of the corollary to [1, Theorem 2] shows that $T(t)$ satisfies (6). This result implies, by virtue of (3), the following inequality:

$$
\left\|X(t) X^{-1}(\tau)\right\| \leqq M e^{-\mu(t-\tau)}, \quad t \geqq \tau \geqq t^{\prime},
$$

for some $M>0$. With (7), a continuity argument then suffices to show that $X(t)$ satisfies (i).

REMARK. It is interesting to note that when $A(t)=A$, a constant matrix, Theorem 1 remains true with the condition reduced to the mere convergence of $Y(t)$ (cf. [2, Theorem XVII]).

III. Consider now the homogeneous system

$$
\frac{d w}{d t}=B(t) w,
$$

where, again, $B(t)$ is an $n \times n$ matrix whose complex-valued elements are uniformly bounded and measurable on the right half-line. The matrix $B(t)$ will be said to be of type $\left(^{*}\right)[3 ; 4]$ if to each constant $n$-vector, $d$, there corresponds a solution, $w(t)$, of (8) for which $\lim w(t)=d$. Type $\left(^{*}\right)$ matrices have the following characterization [4]:

$(\beta) B(t)$ is of type $\left(^{*}\right)$ iff to each nonsingular constant matrix, $C$, there corresponds a fundamental matrix, $W(t ; C)$, of (8) for which $\lim W(t ; C)=C$.

Referring to the defining property for type $(\sim)$ matrices, it is seen that, by choosing $p(t)$ so that $\lim p(t)=Y^{-1}(\infty) c$, every solution of (1) can be made to converge to an arbitrary preassigned constant vector, $c$. Thus type $\left(^{*}\right)$ and type $(\sim)$ matrices are, in this sense, analogues and one anticipates the existence of an even more intimate connection between these two types of matrices. This anticipation is substantiated in the subsequent theorems.

THEOREM 2. If $B(t)$ is of type (*) and if $A(t)=A(t ; C)$, where

$$
A(t ; C)=B(t)+W^{-1}(t ; C),
$$

then for every stability matrix, $C, A(t)$ is of type $(\sim)$ and $Y(\infty)=-C$.

It is a matter of direct verification to discover that $-W(t ; C)$ satisfies the matrix equation

$$
\frac{d Z}{d t}=A(t) Z+I
$$


as does $-X(t) W(0 ; C)+Y(t)$, so that uniqueness implies

$$
Y(t)=X(t) W(0 ; C)-W(t ; C) .
$$

It is apparent that, if $X(t)$ satisfies (i), then $\lim \|X(t)\|=0$ so that we obtain from (11), $Y(\infty)=-C$. The conclusion of the theorem then follows by virtue of $(\alpha)$.

It is thus sufficient to show that $X(t)$ satisfies (i) and, to accomplish this, the device introduced in the proof of Theorem 1 is employed. We define the matrix $Q(t)$ by

$$
Q(t)=W^{-1}(t ; C) X(t)
$$

which may be differentiated to obtain

$$
\frac{d Q}{d t}=W^{-1}(t ; C) Q
$$

This last equation may in turn be written as

$$
\frac{d Q}{d t}=\left[C^{-1}+F(t)\right] Q
$$

where $\lim \|F(t)\|=0$. The argument then proceeds from (12) and (13) in precisely the same manner as in the second part of the proof of Theorem 1, leading to the desired conclusion.!

Theorem 2 has the following partial converse.

Theorem 3. If $A(t)$ is of type $(\sim)$ and if, for some sufficiently large, finite $t^{\prime}, B(t)$ satisfies

$$
B(t)=A(t)+Y^{-1}(t)
$$

on $\left[t^{\prime}, \infty\right)$, then $B(t)$ is of type $\left(^{*}\right)$.

It may be directly verified that the columns of $Y(t)$ satisfy (8) on $\left[t^{\prime}, \infty\right)$; the fundamental matrix $W(t ; Y(\infty))$ which coincides with $Y(t)$ on $\left[t^{\prime}, \infty\right)$ is then found to be $W(t ; Y(\infty))=\tilde{W}(t)\left[\tilde{W}^{-1}\left(t^{\prime}\right) Y\left(t^{\prime}\right)\right]$, where $\tilde{W}(t)$ is that fundamental matrix of $(8)$ for which $\tilde{W}(0)=I$.

We may now prove the following representation theorem, which contains the corollary to [1, Theorem 2] as well as several well-known theorems of stability for the homogeneous equation associated with (1).

THEOREM 4. If

$$
A(t)=A+B(t)+C(t)
$$

where $A$ is a stability matrix, $B(t)$ is of type $\left(^{*}\right)$ and $\lim \|C(t)\|=0$, 
then $A(t)$ is of type $(\sim)$ and $Y(\infty)=-A^{-1}$. Moreover, for sufficiently large $t^{\prime}<\infty$, every $A(t)$ of type $(\sim)$ has a representation of this form on $\left[t^{\prime}, \infty\right)$.

From Theorem 2 we know that the matrix

$$
A+B(t)+\left[W^{-1}\left(t ; A^{-1}\right)-A\right]
$$

is of type $(\sim)$. Since the bracketed term in (15) tends to zero as $t \rightarrow \infty$, it follows by [1, Theorem 2] that $A+B(t)$ is of type $(\sim)$; another application of $[1$, Theorem 2$]$ then establishes the first statement of Theorem 4. For the proof of the second statement, we note that, by virtue of Theorem 3

$$
A(t)=-Y^{-1}(\infty)+B(t)+\left[Y^{-1}(\infty)-Y^{-1}(t)\right], \quad t^{\prime} \leqq t<\infty ;
$$

since Theorem 1 implies that $-Y^{-1}(\infty)$ is a stability matrix, the conclusion is immediate.

Wintner [4] has shown that, in order that $B(t)$ be a type $\left(^{*}\right)$ matrix, it is sufficient (but not necessary) that $B(t)$ satisfy the following condition: $(\gamma) B_{0}(t)=\int_{i}^{\infty} B(\tau) d \tau<\infty$ and either $\int_{\infty}^{\infty}\left\|B(t) B_{0}(t)\right\| d t<\infty$ or $\int \infty\left\|B_{0}(t) B(t)\right\| d t<\infty$.

The ramifications [4] of $(\gamma)$ make it clear that, as stated previously, the corollary to [1, Theorem 2] is contained in Theorem 5. In the scalar case, $n=1$, a simple example of a type $\left(^{*}\right)$ matrix which satisfies neither of the conditions of [1, Theorem 2] is given by $B(t)$ $=\exp i t^{p}, p>1$.

We conclude by answering the question: for a type $(\sim)$ matrix $A(t)$, when does $\lim (d Y / d t)=0$ ?

THEOREM 6. In order that $\lim (d Y / d t)=0$ for a type $(\sim)$ matrix $A(t)$, it is necessary and sufficient that $A(t)$ converge to a stability matrix.

The sufficiency is implied by the corollary to [1, Theorem 2]. For the necessity, we have $A(t)=(d Y / d t-I) Y^{-1}(t)$ for all sufficiently large values of $t$; from this it follows that $\lim A(t)=-Y^{-1}(\infty)$.

\section{REFERENCES}

1. T. F. Bridgland, Jr., A symptotic behavior of the solutions of nonhomogeneous differential equations, Proc. Amer. Math. Soc. 12 (1961), 546-552.

2. - Stability of linear signal transmission systems, SIAM Rev. 5 (1963),

3. A. Wintner, On linear asymptotic equilibria, Amer. J. Math. 71 (1949), 853-858.

4. - On a theorem of Bôcher in the theory of ordinary linear differential equations, Amer. J. Math. 76 (1954), 183-190.

Martin Company, Denver, Colorado 